\title{
Echocardiographic detection of mitral valve aneurysm in patient with infective endocarditis
}

\author{
F ENIA, G CELONA, V FILIPPONE \\ From Divisione di Cardiologia, Ospedale "V Cervello", Palermo, Italy
}

SUMMARY An aneurysm of the anterior mitral valve leaflet was detected on standard M-mode and two dimensional echocardiograms obtained from a patient with infective endocarditis involving the aortic and mitral valves. Two dimensional echocardiography allowed a clear anatomical definition of the aneurysm, confirmed at operation. In this case two dimensional echocardiography was more informative than cardiac catheterisation.

True aneurysms of the mitral valve complicating infective endocarditis are uncommon. ${ }^{12}$

We report an unusual case of aneurysm of the anterior mitral valve leaflet in a patient with infective endocarditis involving the mitral and aortic valves. Echocardiography allowed a clear anatomical

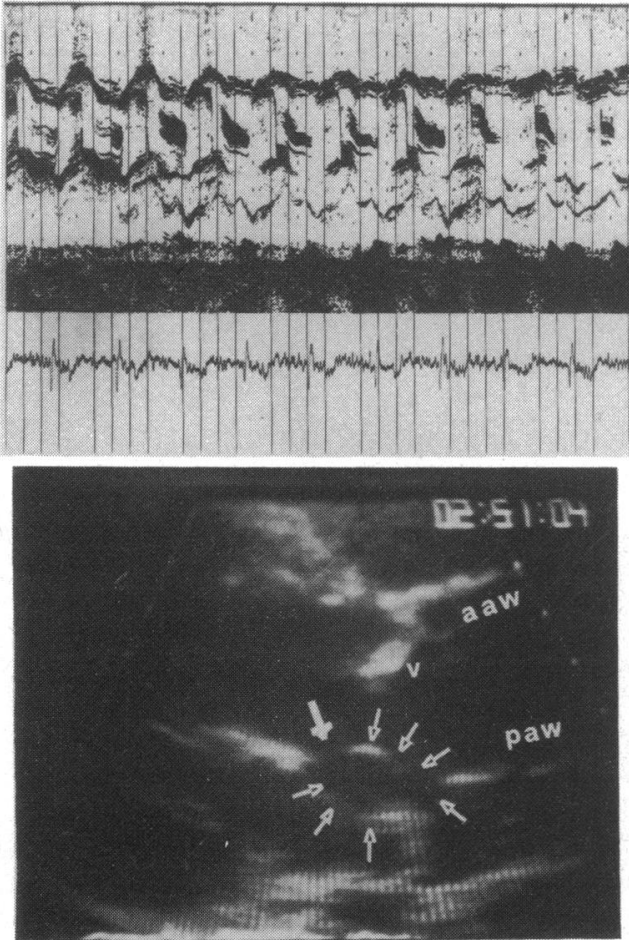

DIASTOLE

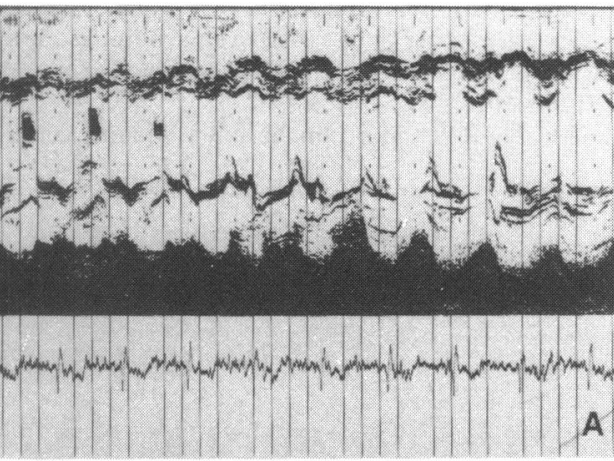

Fig. 1 (A) M-mode echocardiogram showing aortic valve vegetations and an echo free space posterior to the aorta, delimited by an abnormal echo with anterior mitral valve leaflet-like motion. There is aortic mitral discontinuity. (B) Two dimensional echocardiogram (long axis view in systole and in diastole) showing a clear anatomical definition of the aneurysm: the origin (the larger arrow), size, and protrusion into the left atrium. ao, aorta; aaw, anterior aortic wall; paw, posterior aortic wall; la, left atrium; lv, left ventricle; aml, anterior mitral valve leaflet; $v$, vegetation. 
definition of the aneurysm, confirmed at operation.

\section{Case report}

The patient was a 29 year old man, who was well until four months before admission, when there was an abrupt onset of low grade fever, weakness, and arthralgia. On examination the lungs were clear, there was no hepatomegaly, and no peripheral oedema. The spleen was palpable $1 \mathrm{~cm}$ below the left costal margin. A grade $2 / 6$ pansystolic murmur and a third heart sound were audible at the apex. At the left sternal border there was a grade $2 / 6$ ejection systolic murmur and a grade $3 / 6$ early diastolic murmur. The blood pressure was $130 / 60 \mathrm{mmHg}$. Blood cultures grew Streptococcus viridans. The electrocardiogram was normal. The chest $x$-ray film showed a slightly enlarged heart.

M-Mode echocardiography (Fig. 1A) showed aortic valve vegetations with a small echo free space posterior to the aorta at the level of the mitral aortic junction, delimited by an abnormal echo with anterior mitral valve leaflet-like motion behind the posterior aortic wall. Aortic mitral discontinuity was evident.

Two dimensional echocardiography (Fig. 1B) showed a saccular bulging which originated from the anterior mitral valve leaflet and protruded into the left

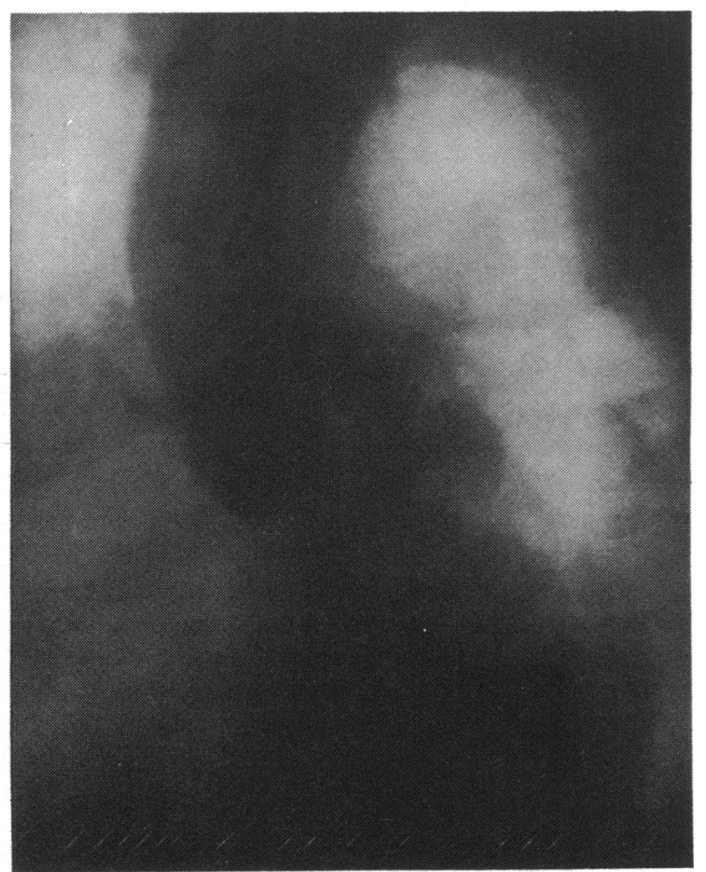

Fig. 2 Cine frame of left anterior oblique aortic root angiogram showing a small saccular collection of contrast medium protruding into the left atrium. atrium. Its wall was separated from the posterior aortic root and its approximate size was $1.5 \times 1 \mathrm{~cm}$. Its motion pattern was similar to that of the anterior mitral valve leaflet with a slight systolic expansion. These data were better appreciated in the long axis parasternal plane. The short axis plane showed an intact aortic root. The echocardiographic diagnosis was possible anterior mitral valve leaflet aneurysm.

Left ventriculography showed mild mitral regurgitation and a slightly dilated but normally contracting left ventricle, and aortography revealed moderate aortic regurgitation. The left anterior oblique projection (Fig. 2) showed a small saccular collection of contrast medium protruding into the left atrium which was interpreted by the radiologist as a possible sinus of Valsalva aneurysm or a possible subaortic annular aneurysm.

At operation the aortic valve was regurgitant and showed vegetations. A small aneurysm arising from the base of the anterior mitral valve leaflet was present. Below the mouth of the aneurysm there were two friable vegetations and a small perforation. The mitral vegetations were easily removed, and the orifice of the aneurysm, and the small perforation, were closed with multiple sutures which were incorporated in the implanted Carpentier aortic prosthesis. The postoperative course was uncomplicated.

\section{Discussion}

It is likely that in this patient the infection of the mitral valve was secondary to the involvement of the aortic valve. Gonzalez-Lavin et $a l .^{3}$ reported secondary involvement of the mitral valve in 10 patients out of 58 operated upon for aortic regurgitation produced by infective endocarditis. The mitral valve may become infected as a complication of aortic valve endocarditis through one of two mechanisms: direct extension of infection along the mitral aortic intervalvular fibrosa up to the anterior mitral valve leaflet, or impingement of a regurgitant stream through the aortic valve or the ventricular surface of the anterior mitral valve leaflet. ${ }^{4}$ The infective process and the subsequent healing may weaken a circumscribed portion of valvular tissue, which, yielding to intracardiac pressure, may form a true aneurysm. ${ }^{5}$

These aneurysms, of saccular shape and often perforated, are commonly situated near the base of the anterior mitral valve leaflet and protrude into the left atrium. Their size varies from 1 to $4 \mathrm{~cm}$ in diameter and they may contain thrombi. ${ }^{1}$

Barack et al. ${ }^{6}$ showed the cineangiographic features of an aneurysm of the anterior mitral valve leaflet. In a steep left anterior oblique projection aneurysms of the anterior mitral valve leaflet are recognised as smooth convex posterior collections of contrast medium 
extending from the anterior mitral valve leaflet and protruding into the left atrium.

In our case, two dimensional echocardiography provided more exact details than cardiac catheterisation. The real nature of the aneurysmal sac was not appreciated at angiography by the radiologist who thought it might have been a sinus of Valsalva aneurysm or a subaortic annular aneurysm. The Mmode echocardiographic findings alone were difficult to interpret. A similar pattern may be the result of superimposition of the aortic root and mitral valve echoes because of undue angulation of the ultrasound probe and lack of lateral resolution of the two structures. Aortic root abscess, left sinus of Valsalva aneurysm, and subaortic annular aneurysm may produce a similar appearance. In these cases, $M$-mode echocardiography shows abnormal echoes behind the posterior aortic wall and sometimes the motion of the echoes suggests the presence of a chamber posterior to the aorta, which expands in systole and empties in diastole. $^{7-10}$ In our case, two dimensional echocardiography gave a clear anatomical definition of the aneurysm and helped to distinguish the lesion from an aortic abscess, a sinus of Valsalva aneurysm, or a subaortic annular aneurysm, all possible complications of infective endocarditis.

Furthermore, according to two dimensional echocardiographic features and surgical data the $\mathrm{M}$-mode findings may be interpreted as follows: the abnormal echo with anterior mitral valve leaflet motion behind the posterior aortic wall originates from the wall of the anterior mitral valve leaflet aneurysm, which retains the mitral valve motion pattern. The echo free space represents the aneurysm.

We conclude that echocardiography may provide valuable information in patients with infective endocarditis and may delineate anterior mitral valve leaflet aneurysms better than invasive investigations.

\section{References}

1 MacLean N, MacDonald MK. Aneurysm of the mitral valve in subacute bacterial endocarditis. Br Heart $\mathcal{F} 1957$; 19: $550-4$.

2 Di Matteo J, Vacheron A, Sabaut D, Delvaux JC, Audoin J. Anévrysmes multiples de la valve mitrale, endocardite bactérienne et syndrome de Marfan. Coeur Med Interne 1971; 10: 519-24.

3 Gonzalez-Lavin L, Lise M, Ross D. The importance of the "jet lesion" in bacterial endocarditis involving the left heart. F Thorac Cardiovasc Surg 1970; 59: 185-92.

4 Edwards JE. Mitral insufficiency secondary to aortic valvular bacterial endocarditis. Circulation 1972; 46: 623 6.

5 English TAH, Honey M, Cleland WP. Ruptured true aneurysm of mitral valve. A complication of aortic valve endocarditis. Br Heart $\mathcal{F}$ 1972; 34: 434-6.

6 Barack BM, Grollman JH Jr, Olinger GN, Levisman JA, MacAlpin RN. Mycotic aneurysm of the mitral valve complicating aortic valve endocarditis. $A \mathcal{F} R$ 1977; 128: 308-10.

7 Mardelli TJ, Ogawa S, Hubbard FE, Dreifus LS, Meixell LL. Cross-sectional echocardiographic detection of aortic ring abscess in bacterial endocarditis. Chest 1978; 74: 576-8.

8 Wong BYS, Bogart DB, Dunn MI. Echocardiographic features of an aneurysm of the left sinus of Valsalva. Chest 1978; 73: 105-7.

9 Alter BR, Treasure RL, Martin HA, Humphrey SH, Murgo JP, McGranahan GM Jr. Echocardiographic detection of a subannular aortic aneurysm. Am Heart $\mathcal{f}$ 1978; 96: 525-8.

10 Griffiths BE, Petch MC, English TAH. Echocardiographic detection of subvalvar aortic root aneurysm extending to mitral valve annulus as complication of aortic valve endocarditis. Br Heart $\mathcal{F} 1982 ; 47$ : 392-6.

Requests for reprints to Dr Francesco Enia, Via F Liszt 47, 90145 Palermo, Italy. 